\title{
Antimicrobial Prescription Analysis in Relation with Rationality and Cost Effectiveness in a Tropical Diseases Hospital of Eastern India
}

\author{
Santanu Munshi ${ }^{1}$, Debashis Roy Burman ${ }^{2}$, Debkumar Ray $^{3}$, \\ Indranil Dhar ${ }^{4}$, Netai Pramanick ${ }^{5}$ \\ ${ }^{1}$ Professor, Department of Clinical \& Experimental Pharmacology, School of Tropical Medicine, Kolkata \\ ${ }^{2}$ AssistantProfessor, Department of Oncopathology, Medical College, Kolkata \\ ${ }^{3}$ Assistant Professor, Department of Biochemistry, BankuraSammilani Medical College, \\ Bankura \\ ${ }^{4}$ Assistant Professor Department of Laboratory Medicine School of Tropical Medicine, Kolkata \\ ${ }^{5}$ Assistant Professor Department of Tropical Medicine, School of Tropical Medicine, Kolkata
}

\begin{abstract}
:
Objectives: Antimicrobials are the most commonly used and at the same time misused drugs in prescriptions. The present pilot survey in a government teaching hospital of tropical diseases has been targeted to generate the utilization data of antimicrobial agents based on which a specific Institutional antimicrobial guideline could be generated.

Methods: 100 prescriptions, each containing at least one or more antimicrobial agents were collected in a random sampling manner from the medical indoor of Carmichael Hospital for Tropical Diseases. Assessment of rationality of all prescriptions was performed.

Results: The present study showed that out of 100 prescriptions $8 \%$ of the prescriptions were semi-rational, $1 \%$ was irrational and the rest were considered rational. Among the different classes of antimicrobial agents cephalosporins remained the most frequently (33\%) prescribed agent followed by aminopenicillins (15\%).

Discussion: Very low incidence of semirational and irrational prescriptions in this pilot study is a good indicator of proficiency of the prescribers and availability of good hospital supply. Polypharmacy was noted in large no. of prescriptions having some associated tropical diseases, especially in multiple infections. In most of the cases first choice antimicrobials were used.

Conclusions: For rational use of antimicrobials more importance should be given on laboratory confirmation by culture sensitivity tests of different clinical specimens. Further study is necessary to formulate a standard antimicrobial guideline for common infective ailments in hospital practice of tropical diseases.
\end{abstract}

Key words: antimicrobials, cost analysis, prescription audit, tropical disease, tertiary hospital.

\section{Introduction}

Infectious diseases keep people in poverty. Worm infestation and respiratory diseases are common in poor developing countries. The big three infectious diseases-HIV/AIDS, TB and malaria claimed 5.7 million lives worldwide in 2001. Ongoing ill health is a major reason why the poor are not able to break out of the cycle of poverty. Infections leads to poverty, poverty leads to infections. ${ }^{[1]}$

Antimicrobials are most commonly used and at the same time misused drugs in prescriptions. Widespread irrational usage of antimicrobials, shortage of antimicrobial supply in government hospitals, low purchasing capacity of patients and incidences of antimicrobial resistance complicate the outcome of therapies. ${ }^{[2]}$ Use of restricted antibiotic guidelines will be a step towards the up gradation in the antibiotic policies of many multispeciality and tertiary healthcare centers, which will help reduce antibiotic resistance in the long run. ${ }^{[3]}$ Although several documentations of antibiotic resistance and the spread of resistant strains within hospitals and the community have been published and continue to be reported from India, utilization of antibiotics for patients with associated tropical diseases are not published.

Moreover, immunocompromised state is a predisposing factor for tropical infectionsasit complicates the sensitivity pattern to antimicrobials. The present pilot survey in a government teaching hospital of tropical diseases has been targeted to generate the utilization data of antimicrobial agents based on which a specific Institutional antimicrobial guideline could be generated. 


\section{Materials\& Methods}

100 prescriptions, each containing at least one or more antimicrobial agents were collected in a random sampling manner from the medical indoor of Carmichael Hospital for Tropical Diseases. Prescriptions containing antimicrobials of any category were selected irrespective of ailments, age, or sex of the patients or the route of administration of the drug. The antimicrobials studied included the prescriptions made for common ailments like upper respiratory tract infections, lower respiratory tract infections, urinary tract infections, gastrointestinal tract infections and others, which included skin and soft tissue infections, CNS infections and malaria. Provisional diagnosis made in each case was also recorded. Any change in antimicrobial prescription according to the results of laboratory investigations was recorded. Assessment of rationality of all prescriptions was performed. ${ }^{[4]}$

Maximum 30 marks were assigned per prescription and distributed as follows $-50 \%$ of the marks for the correctness of the drug selected and the rest for its correctness of dose and duration of the therapy. In case of multiple antimicrobials, 20 marks were assigned for the main drug/drugs and 10 marks for any complimentary drug/drugs, which in each case were redistributed as above. ${ }^{[3]}$ The appropriateness of drug selected and its correctness in respect to the dose and duration of therapy were assessed as per standard drug treatment guideline.

Negative marks were assigned for use of inappropriate drug (-5), hazardous drug (-5), unnecessary drug $(-5)$, and grossly irrational drug (-10). Every prescription was graded according to the marks obtained on the basis of following criteria- upto 14 marks- irrational, 15-24 marks-semi-rational, 25-30 marks-rational. The rationality of use of antimicrobials in accordance with provisional diagnosis, the number of antimicrobials used per prescription and cost of each prescription were analysed. ${ }^{[5]}$ The cost calculation of total duration of antimicrobial therapy per prescription was performed on the basis of current prevailing market as depicted in 'Drug Today'.

\section{Results}

The present study showed that 191 antimicrobials were used for 100 prescriptions. Out of these $56 \%$ of cases were cured, $29 \%$ improved and theremaining $13 \%$ were treatment failure. Again $8 \%$ of the prescriptions were semi-rational, $1 \%$ irrational and the rest $(91 \%)$ were considered rational. The prescriptions containing single antimicrobial were the most common (46\%), followed by those containing two antimicrobials (32\%) and $22 \%$ of prescriptions contained more than two agents.In all the prescriptions $33 \%$ of drugs were written in generic name and rest of the drugs in 'brand' name. Orally used drugs were $40 \%$ and those used topically were $4.7 \%$ rest of the drugs used parenterally (either im or iv). It was observed that the antimicrobials in accordance with the provisional diagnosis was most rational (100\%) in skin and soft tissue infections, followed by gastrointestinal tract infection (95\%), respiratory tract infection and urinary tract infection $85 \%$ each while it was minimum (80\%) in other infections.(fig.1)

Among the different classes of antimicrobials agents cephalosporins remained the most frequently (33\%) prescribed agent followed by aminopenicillins (15\%), quinolones (12.5\%) and macrolides $(12.5 \%)$. (fig.2)

Demographic distribution showed that the antimicrobials prescribed were most rational (94\%) in the age group 41-60, while it was minimum (87.5\%) in the age group 61-80.(fig.3)

It was further observed that the cost per prescriptionfor the total duration of antimicrobial treatment was below Rs. 250 in only 32\% cases while the remaining 68\% showed variation in the cost (Rs.250 to Rs.5000). Another 50\% of the prescriptions increased a cost Rs. 250 to 500 for the total amount of treatment, while $29 \%$ increased a case of Rs 1000- 1500 and remaining 24\% had to spend more than Rs 1500. Mean cost of drugs per prescription is Rs.450 and mean duration of each prescription is 7 days. It was observed that only 33drugs $(17.3 \%)$ were prescribed from the Essential Drug list of India.

Figure 1.Rationality of antimicrobial usage in different diseases expressed in percentile

\begin{tabular}{|l|c|c|c|c|c|}
\hline Disease & RTI & GITI & UTI & SSTI & OTHERS \\
\hline Semirational & 15 & 5 & 15 & 0 & 20 \\
\hline Rational & 85 & 95 & 85 & 100 & 80 \\
\hline & & & & & \\
\hline
\end{tabular}




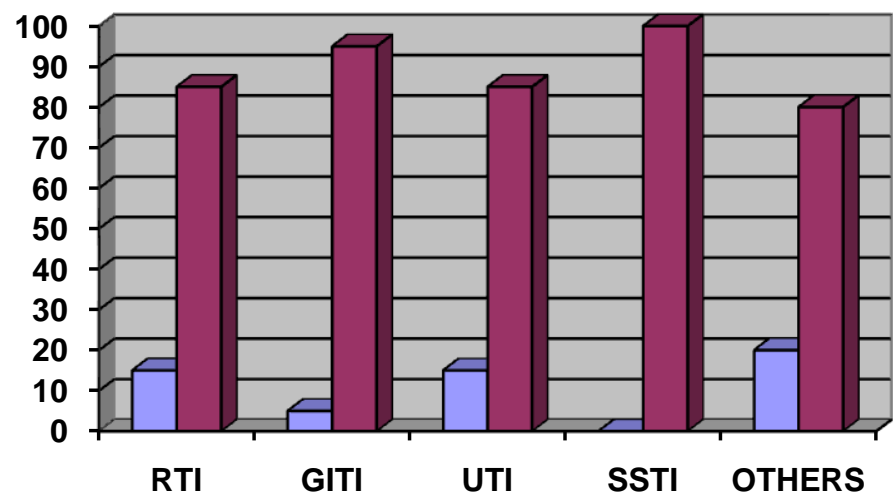

\section{口Semirational}

口Rational

Figure 2.Frequency of different classes of antimicrobials prescribed expressed in Percentile

\begin{tabular}{|l|l|l|l|l|l|l|l|l|l|l|l|}
\hline AMA & $\begin{array}{l}\text { Amino } \\
\text { penicillin }\end{array}$ & $\begin{array}{l}\text { Amoxy } \\
\text {-clav }\end{array}$ & $\begin{array}{l}\text { Cepha } \\
\text { losporin }\end{array}$ & $\begin{array}{l}\text { Quin } \\
\text { olone }\end{array}$ & $\begin{array}{l}\text { Nitroimi } \\
\text { dazole }\end{array}$ & ATD & $\begin{array}{l}\text { Aminoglyc } \\
\text { osides }\end{array}$ & $\begin{array}{l}\text { Sulfonami } \\
\text { des }\end{array}$ & $\begin{array}{l}\text { Mac } \\
\text { rolide }\end{array}$ & $\begin{array}{l}\text { Tetra/ } \\
\text { Doxy }\end{array}$ & thers \\
\hline$\%$ & $\mathbf{1 2}$ & $\mathbf{3}$ & $\mathbf{3 3}$ & $\mathbf{1 2 . 5}$ & $\mathbf{8 . 9}$ & $\mathbf{1}$ & $\mathbf{5 . 1}$ & $\mathbf{1}$ & $\mathbf{1 2 . 5}$ & $\mathbf{4}$ & $\mathbf{7 . 3}$ \\
\hline
\end{tabular}

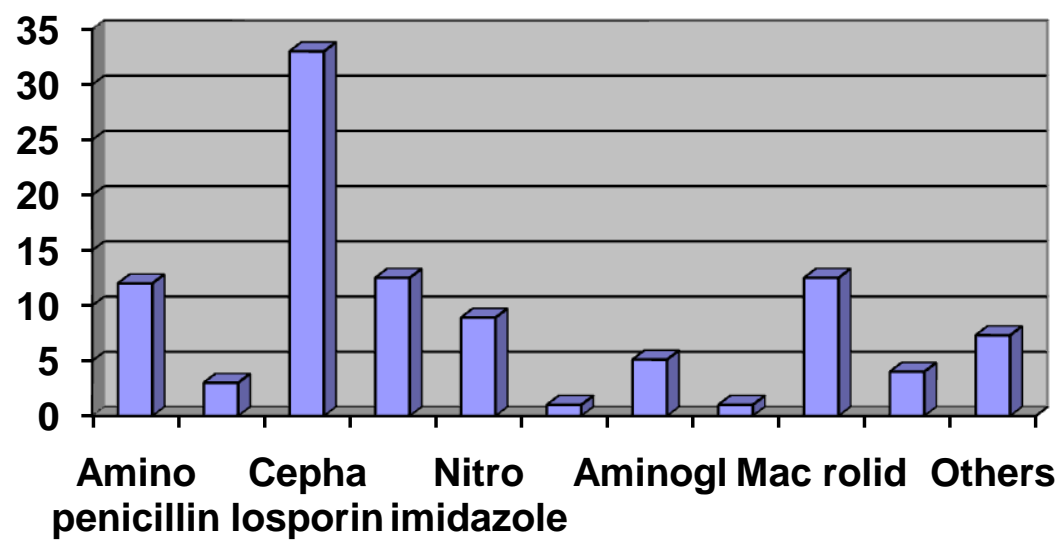

Figure 3. Frequency of distribution of antimicrobials prescribed in different age groups expressed in percentile

\begin{tabular}{|c|c|c|c|c|c|}
\hline Age gr. & $0-20 \mathrm{yrs}$ & $21-40 \mathrm{yrs}$ & $42-60 \mathrm{yrs}$ & $61-80 \mathrm{yrs}$ & $81-100 \mathrm{yrs}$ \\
\hline $\mathbf{\%}$ & $\mathbf{2 9}$ & $\mathbf{3 3}$ & $\mathbf{2 9}$ & $\mathbf{8}$ & $\mathbf{1}$ \\
\hline
\end{tabular}

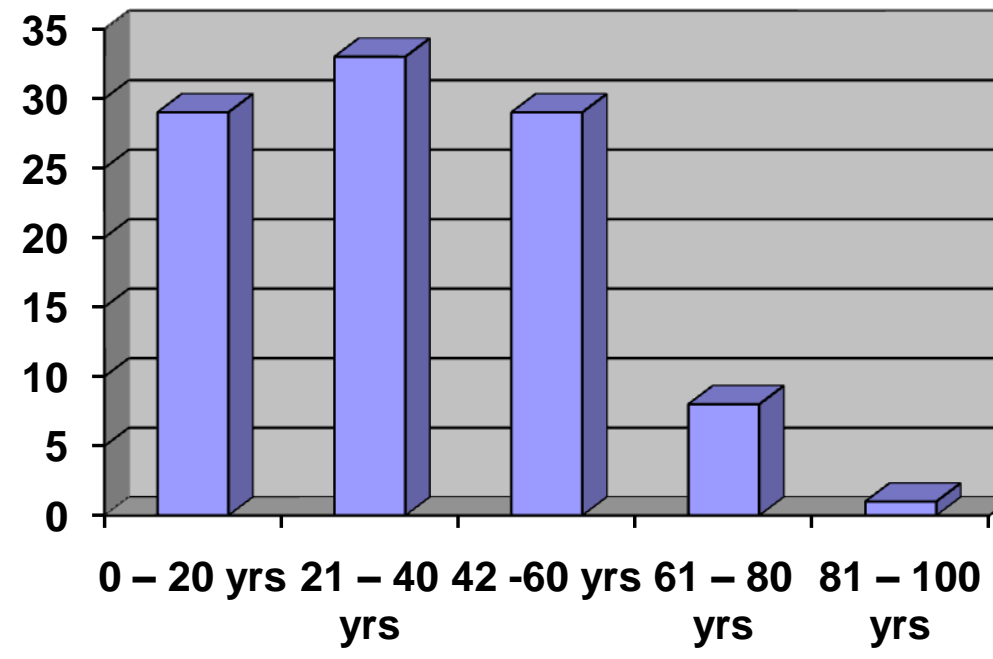




\section{Discussions}

The semirationality or irrationality of the use of antimicrobials could be attributed to the use of multiple antimicrobials, non-use of first choice antimicrobials on the basis of provisional diagnosis, and the use of irrational and unnecessary antimicrobials. Very few no. of such cases in this pilot study is a good indicator of proficiency of the prescribers and availability of good hospital supply. ${ }^{[6]}$ The polypharmacy of antimicrobials observed in the prescription may be due to empirical uses of antimicrobials without corroborative culture and sensitivity test. Some of the prescriptions follow the recommended polypharmacy for the particular ailment. Also it was seen that most of the drug were not selected from the Essential Drug listof India and there was an increasing trend of using costly parenteral drugs. Mean cost of therapy was also observed to be high in comparison to other similar antibiotic prescription audits. As many of the patients were having some associated tropical diseases, polypharmacy with the use of costly parenteral antibiotics were noted in many prescriptionsespecially those in multiple infections. In most of the cases first choice antimicrobials were used. Surprisingly quinolones were prescribed rationally as compared to previous studies in teaching hospitals. ${ }^{[5][7]}$ Antimicrobialswhich are used restrictedly now-a-days are also found to be prescribed for appropriate ailments, e.g. chloramphenicol in a case of enteric fever (diagnosed with rising titre in Widal test) not responding to cephalosporins. Tetracycline was given for liver abscess along with metrogyl, Doxycycline usedin combination with metrogyl for liver abscess and in resistant falciparum malaria. Tetracycline/ Doxycycline and metrogyl were always used along with other animmicrobials and never singly. Linezolid was prescribed for septicaemia.New and more expensive drugs like moxifloxacin have been used for respiratory tract infection. This study shows a good response of oral amoxycillin for URTI in hospital admitted patients. For rational use of antimicrobials more importance should be given on laboratory confirmation by culture sensitivity tests of different clinical specimens. ${ }^{[8][9]}$ As immunocompromised state is a predisposing factor for tropical infections, duration of treatment and hospital stay is longer than usual for many patients.

\section{Conclusion}

Variation in cost analysis warrants the existence of standard antimicrobial guidelines for common infective ailments in hospital practice. Development of an in-house IV-to-Oral switchover guideline and dissemination of the knowledge through education and training to concerned persons will help reduce the cost and avoid side effects. ${ }^{[10]}$ Further studies are proposed to review the cost-effectiveness and correlation existing between all above mentioned parameters.

\section{Acknowledgement}

To the Director, School of Tropical Medicine, Kolkata for permitting to undertake this study.

\section{References:}

[1] World Health Organization. Scaling up the response to infectious diseases - A way out of poverty. Report on infectious diseases 2002. WHO/CDS/2002.7. Geneva: 2002.

[2] Kumari Indira K.S, S. J. Chandy, L. Jeyaseelan, Rashmi Kumar and Saradha Suresh. Antimicrobial prescription patterns for common acute infections in some rural \& urban health facilities of India. Indian J Med Res 2008; 128: 165-171.

[3] Anita Kotwani, Kathleen Holloway and R.R. Chaudhury. Methodology for surveillance of antimicrobials use among out-patients in Delhi. Indian J Med Res 2009; 129: 555-560.

[4] Summers RS. Drug utilization in internal medicine wards at a teaching hospital serving a developing community. S Afr Med J 1985; 67:549-52.

[5] Das AK, Roy K, Kundu KK, Das N, Munshi S. Study of rational utilization and cosl analysis of antimicrobials in a government teaching hospital. Ind J Pharmacol 2002; 34:59-61.

[6] McCaffertyJA,and Lang SDR. An audit of restricted antibiotic use in a general hospital. N Z Med J 1988; 101: 210-211.

[7] Rajesh Bhatia and Jai P. Narain, The growing challenge of antimicrobial resistance in the South-East Asia Region - Are we losing the battle? Indian J Med Res 2010;132: 482-486.

[8] RanjeetaKumari, M. Z. Idris, VidyaBhushan, AnishKhanna, Monika Agrawal, and Shivendra Kumar Singh. Assessment of prescription pattern at the public health facilities of Lucknow district. Indian J Pharmacol 2008; 40(6): 243-247.

[9] Victorian Postgraduate Foundation Inc. Antibiotic Guidline Australia. The Foundation, 1990.

[10] Viswanathan N, Gandhi IS, Shashindran CH, Aithan C. Drug utilization study of antimicrobial agents. Indian J Med Res 1981; 74:772-8. 